\title{
Efficacy and safety of endostatin in combination with chemotherapy in small cell lung cancer: a phase 2 single-arm multicenter open-label trial
}

\author{
Yi Zhao" ${ }^{1 \#}$ Xu Zhang ${ }^{2 \#}$, Chenxing Jin ${ }^{1 \#}$, Xiaomin $\mathrm{Yu}^{3}$, Min Zhang ${ }^{4}$, Yang Cao ${ }^{5}$, Ying Li $^{1}$, Aman Wang ${ }^{1}$, \\ Xiu Shan ${ }^{1}$, Jia Zhang ${ }^{6}$, Juhong Wang ${ }^{1}$, Liu Yin ${ }^{1}$, Xiaoxin Tan ${ }^{1}$, Jiwei Liu ${ }^{1}$ \\ ${ }^{1}$ Department of Oncology, The First Affiliated Hospital of Dalian Medical University, Dalian, China; ${ }^{2}$ The Clinical Skill Training Center of Dalian \\ Medical University, Dalian, China; ${ }^{3}$ Department of Oncology, The Central Hospital of Dalian, Dalian, China; ${ }^{4}$ Department of Oncology, Central \\ Hospital, Pulandian District, Dalian, China; ${ }^{5}$ Department of Oncology, The 967th Hospital of Joint Logistics Force, Dalian, China; ${ }^{6}$ Department of \\ Oncology, The People's Hospital of Ningxiang City, Ningxiang, China \\ Contributions: (I) Conception and design: Y Zhao, J Liu; (II) Administrative support: Y Zhao, J Liu; (III) Provision of study materials or patients: Y \\ Zhao, X Yu, M Zhang, L Yin, X Tan; (IV) Collection and assembly of data: J Zhang, C Jin; (V) Data analysis and interpretation: C Jin, A Wang, Y Li; \\ (VI) Manuscript writing: All authors; (VII) Final approval of manuscript: All authors. \\ "These authors contributed equally to this work and should be considered as co-first authors. \\ Correspondence to: Jiwei Liu, MD; Xiaoxin Tan, MM. Department of Oncology, The First Affiliated Hospital of Dalian Medical University, Dalian, \\ Liaoning, Zhongshan Road 222, Dalian 116011, China Email: jiweiliudl@126.com; txxd12007@126.com.
}

Background: Small-cell lung cancer (SCLC) is a type of lung cancer with high invasiveness and poor prognosis. Although SCLC is effective for initial treatment, the vast majority of patients will relapse, the efficacy of posterior line therapy is limited, and there is a lack of effective treatment. At the same time, in the past 30 years, there has been little progress in first-line treatment. With the progress of antiangiogenic therapy, whether it can be used in the treatment of SCLC is worth exploring. Therefore, a single-arm multicenter clinical study was conducted on the efficacy, optimization, and safety of endostatin combined chemotherapy in SCLC.

Methods: This study is a prospective non-blind single-arm multicenter study. From January 2016 to July 2019, a total of 22 patients with histologically diagnosed SCLC were enrolled in three centers. The treatment regimen was as follows: continuous intravenous pump infusion of endostatin (90 mg) for 72 hours, 3 days before chemotherapy, and continuous pump infusion of endostatin $(120 \mathrm{mg})$ for 96 hours the next day following the infusion of chemotherapeutic drugs; the chemotherapy regimen was administered with standard platinum combined with etoposide once every 21 days. After six cycles, endostatin maintenance therapy was used until the disease progressed or intolerable adverse reactions occurred. The therapeutic effect was evaluated by imaging and oncology markers every two cycles, and the adverse reactions, tumor progression time, and patient survival time were recorded.

Results: Among the 21 patients analyzed, the median progression-free survival (PFS) was 8.0 months, the median overall survival (OS) was 13.6 months, the objective effective rate (ORR) was $61.9 \%$, and the disease control rate (DCR) was $95.2 \%$. All patients tolerated the treatment. The main adverse reactions were myelosuppression, albuminuria, nausea, and vomiting. The incidence of grade 3 or 4 adverse reactions was $7.2 \%$, which could be relieved by symptomatic support treatment. There were no treatment-related deaths. Conclusions: Endostatin combined with platinum-etoposide is safe and effective in the treatment of SCLC.

Keywords: Small cell lung cancer (SCLC); endostatin; antiangiogenic therapy; chemotherapy; clinical trail

Submitted Jan 21, 2021. Accepted for publication Mar 25, 2021.

doi: 10.21037/apm-21-443

View this article at: http://dx.doi.org/10.21037/apm-21-443 


\section{Introduction}

Lung cancer is a malignant tumor with high rates of morbidity and mortality both in China and globally. Small cell lung cancer (SCLC) accounts for about $13-17 \%$ of all lung cancers. Due to tobacco exposure, SCLC has a high somatic mutation rate and produces a variety of new antigens, resulting in a higher degree of malignancy and heterogeneity, and is more prone to local infiltration and distant metastasis. At the time of diagnosis, between $60-70 \%$ of patients are already in the extensive-stage (ES), and low-dose CT does not show advantages in early diagnosis. Although SCLC is sensitive to radiotherapy and chemotherapy, relapse occurs easily, and the prognosis is poor. The median survival time of ES-SCLC patients is only 7-12 months, and the overall 5 -year survival rate is only $2.8-7.2 \%$, which is not significantly improved compared with the previous (1-5). At present, simultaneous radiotherapy and chemotherapy are commonly used in the clinical treatment of SCLC. For patients who cannot tolerate simultaneous radiotherapy and chemotherapy, sequential radiotherapy and chemotherapy can be utilized (6). Platinum-etoposide is often used as a firstline chemotherapy regimen. In 2017, WCLC statistics showed that $19 \%$ of ES-SCLC patients received secondline treatment after first-line chemotherapy, and the regimen was often platinum combined with etoposide, irinotecan, or topotecan. However, the effect of second-line chemotherapy after the recurrence of platinum-resistant type was unsatisfactory (7).

With immunotherapy, targeted therapy, anti-angiogenic therapy, and other methods to achieve a certain effect in other tumors, strategies that apply these treatments to SCLC have become a particular research focus in order to improve the prognosis of patients. It is well known that the combination of vascular endothelial growth factor (VEGF) and its receptor (VEGFR) can promote angiogenesis, affect the production of oxygen and nutrients in the tumor microenvironment by restoring poor vascularization in the tumor area, and promote the formation of the immunosuppressive environment $(8,9)$. The VEGF family is composed of VEGF A-E and VEGFR 1-3, which can mediate tumor angiogenesis by promoting endothelial cell proliferation, migration, and invasion, and the high expression of VEGF is related to tumor stage, disease progression, chemotherapy resistance, and poor prognosis (10). Endostatin has been independently developed in China, and can not only inhibit tumor angiogenesis, but also normalize abnormal blood vessels in the tumor microenvironment, thus improving hypoxia and increasing the drug concentration in the tumor microenvironment. Furthermore, endostatin can also impede the activation of VEGF 3 by inhibiting the expression of VEGFR C and D in tumor tissue or suppressing its signal transduction, ultimately inhibiting tumor lymphangiogenesis and lymphatic metastasis. When combined with cisplatin, it has a concentration-dependent killing effect on circulating tumor cells (11). Previous experimental data has shown that anti-angiogenic therapy combined with chemotherapy can achieve certain benefits for both PFS and OS. Therefore, we aim to explore whether endostatin combined with chemotherapy can be used successively, and to innovate and optimize the mode of endostatin administration, and to pump continuously instead of traditional intravenous drip before and after chemotherapy, to normalize the blood vessels in the tumor microenvironment, so as to maximize the killing of tumor cells and achieve better clinical outcomes. We performed this experiment to determine the optimal scheme and corresponding efficacy of endostatin combined chemotherapy in order to provide a new basis and novel idea for the treatment of SCLC.

We present the following article in accordance with the TREND reporting checklist (available at http://dx.doi. org/10.21037/apm-21-443).

\section{Methods}

\section{Patients}

From January 2016 to July 2019, 22 patients with histologically or cytologically confirmed treatment-naïve SCLC were enrolled in this interventional clinical trial. All patients were between 18 and 75 years old with an Eastern Cooperative Oncology Group (ECOG) performance status score of 0 to 2, and an expected survival of more than 12 weeks. All patients had at least one measurable disease as per the Response Evaluation Criteria in Solid Tumors (RECIST, version 1.1) criteria. Patients with serious liver and kidney function abnormalities, or those that had serious abnormal blood system results [absolute neutrophil count (ANC)] $\leq 1.5 \times 10^{9} / \mathrm{L}$, platelet count $\leq 100 \times 10^{9} / \mathrm{L}$, and hemoglobin $\leq 9 \mathrm{~g} / \mathrm{dL}$ ) were excluded. The exclusion criteria also included the following: (I) patients with intractable hypertension, cardiovascular, or cerebrovascular diseases; (II) patients with coagulation disorders or bleeding tendency; (III) patients with uncontrollable acute or chronic infection, or prolonged wounds; (IV) those with poor compliance, were uncooperative, or cases where the 


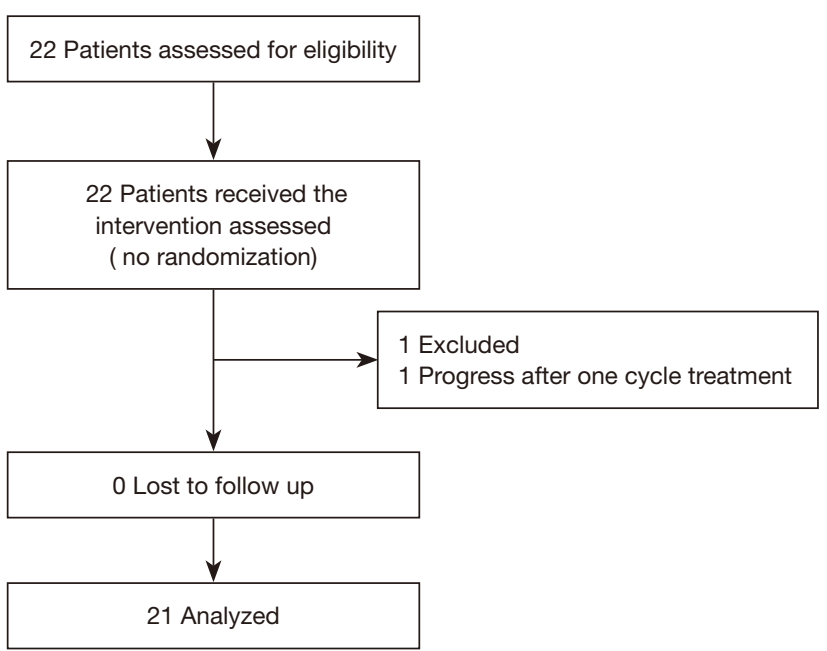

Figure 1 CONSORT diagram.

treatment could not be described to the patient; (V) patients with brain metastases, significant intracranial hypertension, or other mental or neurological diseases; and (VI) patients with other tumors or serious diseases. After review and approval by the hospital ethics committee, a total of 22 patients were finally enrolled in this study. All of the enrolled patients or their family members signed the informed consent and could actively cooperate with the treatment. All procedures performed in this study involving human participants were in accordance with the Declaration of Helsinki (as revised in 2013). The study was approved by ethics committee of First Affiliated Hospital of Dalian Medical University (No. YJ-KYFB-2020-23).

\section{Intervention measures}

The following treatment regimen was given to all patients. Endostatin $(90 \mathrm{mg}$ ) was administered intravenously via a PICC or intravenous infusion port for 72 hours, 3 days before chemotherapy. This was followed by platinumetoposide, and the specific regimen was as follows: etoposide $100 \mathrm{mg} / \mathrm{m}^{2}$, days $1-3$; cisplatin $75-80 \mathrm{mg} / \mathrm{m}^{2}$, day 1 ; or carboplatin: AUC 5-6, day 1. On the second day after the infusion of the chemotherapy drugs, endostatin $(120 \mathrm{mg})$ was continuously injected for 96 hours. Each treatment cycle lasted 21 days, for a total of 4-6 cycles, followed by endostatin maintenance therapy until disease progression or the occurrence of intolerable adverse reactions. The corresponding drug dose was adjusted according to hematological or non-hematological toxicity during the course of treatment and prompt symptomatic support treatment was given. Treatment was ceased as soon as lifethreatening toxic reactions occurred.

\section{Toxic effect assessments}

Evaluations for treatment-associated toxic effects occurred on day 1 and day 13 of every cycle. The National Cancer Institute Common Terminology Criteria for Adverse Events (version 4.0) was used to grade toxic effects. The patients' blood pressure was monitored weekly during treatment, and routine blood and urine, liver and kidney function, oncology markers, electrocardiogram, and other tests were performed during each treatment cycle.

\section{Response assessments}

Efficacy was evaluated by chest CT imaging examination after every two cycles of treatment. The WHO solid tumor efficacy evaluation criteria (RECIST1.1) was used as the evaluation criteria, and included complete response (CR); partial mitigation (PR); disease progression (PD); stable disease (SD); objective effective rate (ORR), and disease control rate (DCR). The prognosis was assessed based on the time to tumor progression and survival during followup, including PFS and OS.

\section{Statistical methods}

SPSS (version 23.0) was used for statistical analysis of the data. The Kaplan-Meier method was used to analyze the PFS and OS of patients in this experiment. GraphPad 6.2 was used to draw the survival curve, and the waterfall diagram was used to reflect the objective situation of clinical efficacy.

\section{Results}

\section{General information}

A total of 22 patients were included in this study. One case was excluded due to the progress after one cycle of treatment. Finally, 21 cases were included in the analysis (Figure 1), and the basic clinical data of all patients are shown in Table 1.

\section{Analysis of short-term clinical effect on patients}

All 22 patients were newly diagnosed with SCLC and had 
Table 1 Baseline characteristics of SCLC patients

\begin{tabular}{lc}
\hline Characteristic & Patients, N=21 (\%) \\
\hline Age, median (range), years & $65[53-79]$ \\
Sex & $9(42.9)$ \\
Female & $12(57.1)$ \\
Male & \\
ECOG & $21(100.0)$ \\
$0-1$ & $0(0)$ \\
$3-4$ & \\
Smoking status & $12(57.1)$ \\
Former, pack-years & $9(42.9)$ \\
Never & \\
Disease stage & $10(47.6)$ \\
LD-SCLC & $11(52.4)$ \\
ED-SCLC
\end{tabular}

SCLC, small cell lung cancer; ECOG, Eastern Cooperative Oncology Group.

Table 2 Therapeutic effect evaluation of SCLC patients

\begin{tabular}{lc}
\hline Therapeutic effect & Patients, N=21 (\%) \\
\hline CR & $4(19.0)$ \\
PR & $9(42.9)$ \\
SD & $7(33.3)$ \\
PD & $1(4.8)$ \\
\hline
\end{tabular}

SCLC, small cell lung cancer; CR, complete response; PR, partial response; SD, stable disease; PD, progression disease.

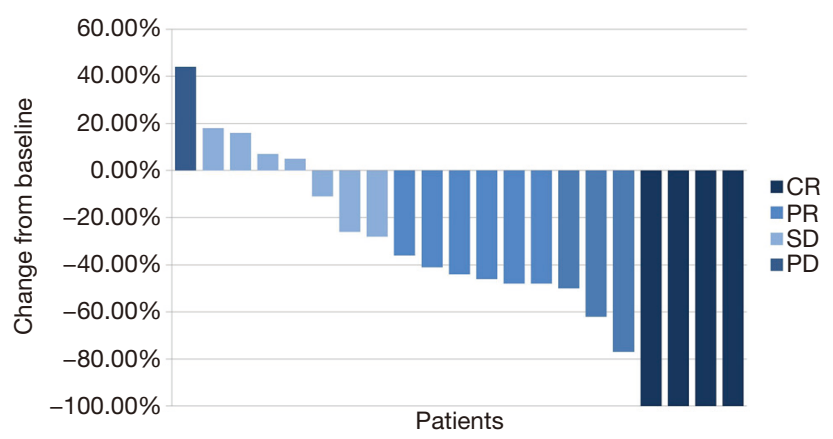

Figure 2 Best overall response. CR, complete response; PR, partial response; $\mathrm{SD}$, stable disease; $\mathrm{PD}$, progression disease.

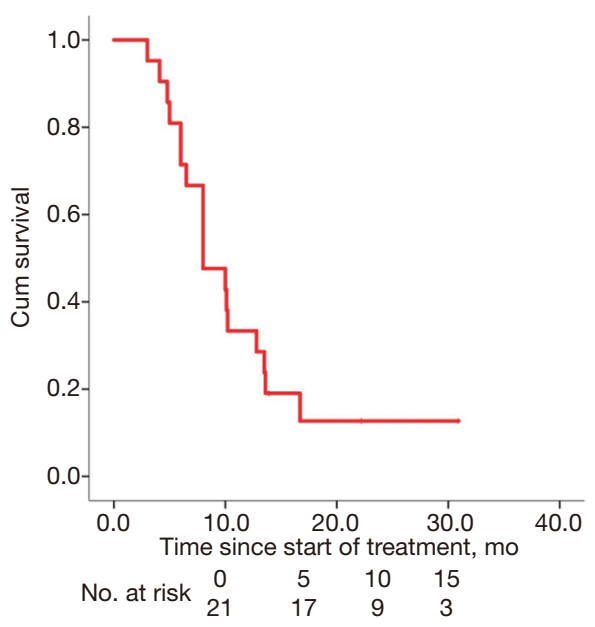

Figure 3 PFS of SCLC patients. PFS, progression-free survival; SCLC, small cell lung cancer.

not received systematic treatment previously. $57.1 \%$ of the patients received endostatin combined with chemotherapy for six cycles, and a total of seven cases $(33.3 \%)$ received endostatin intravenous infusion maintenance therapy. All patients were evaluated by imaging, and the best curative effect after treatment was four cases of CR, nine cases of $\mathrm{PR}$, seven cases of SD, and one case of PD (Table 2). The ORR of this study was $61.9 \%$ and the DCR was $95.2 \%$. The waterfall diagram of the best effect of the 21 patients is shown in Figure 2.

\section{Prognosis}

In terms of prognosis, as of August 31, 2020, six patients were still alive and seven patients had received maintenance therapy. The longest PFS was 30.9 months. The median PFS of the 21 patients was 8 months, and the median OS was 13.6 months. PFS, OS, and the survival curves are shown in Figures 3, 4, and 5, respectively. A total of 11 patients were treated with simultaneous radiotherapy and chemotherapy.

\section{Toxic effects}

This study primarily recorded the occurrence of grade 2 and above adverse reactions to the endostatin combined with chemotherapy regimen (Table 3). The incidence of grade 3 or 4 adverse reactions was $7.4 \%$, and mainly involved 
myelosuppression, such as leukopenia and thrombocytopenia.

\section{Discussion}

A total of 22 newly diagnosed SCLC patients were included in this study. Prognosis data were obtained by imaging evaluation and late follow-up, and the adverse reactions

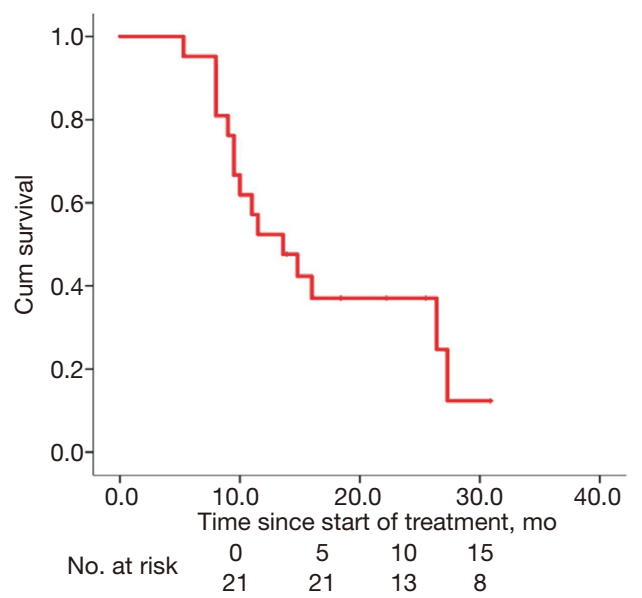

Figure 4 OS of SCLC patients. OS, overall survival; SCLC, small cell lung cancer. during the treatment were evaluated by CTCAE v4.0. Finally, the data of 21 patients were statistically analyzed to explore the clinical efficacy and safety of the optimized "sandwich method" intravenous pump infusion of endostatin combined with chemotherapy in the treatment of SCLC.

SCLC is a high-level neuroendocrine tumor that mainly occurs in smoking patients, accounting for about $15 \%$ of all lung cancer patients; about 250,000 new SCLC occur worldwide each year, causing at least 200,000 deaths. Men are more likely to develop SCLC, but the prevalence rate in women has increased year by year over the past 50 years. Compared with other solid tumors, the mortality rate of SCLC is extremely high. Although the 5-year survival rate of SCLC has improved slightly, the median survival rate between 1983 and 2012 is only 7 months, and the 5 -year survival rate is less than $5 \%$ (12), according to the SEER database. The pathology of SCLC is divided into two subtypes: simple type $(80 \%)$ and mixed type $(20 \%)$. The most common histological subtypes of non-small cell lung cancer in mixed SCLC are large cell carcinoma or large cell neuroendocrine carcinoma, accounting for $4-16 \%$ of SCLC, while other mixed SCLC accounts for only $1-3 \%$ of all SCLC. The clinical manifestations, chemotherapy efficacy, and survival rate of patients with mixed SCLC were similar

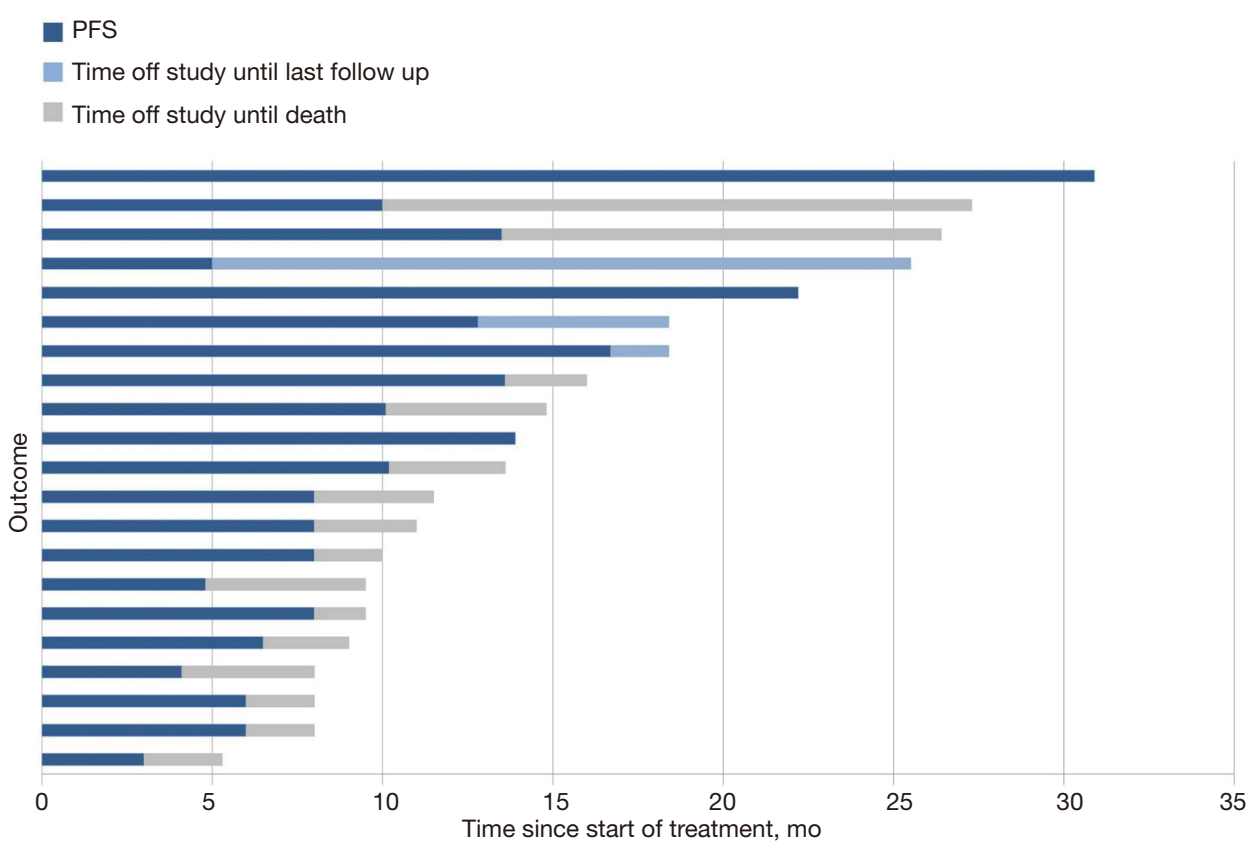

Figure 5 PFS and OS of SCLC patients. PFS, progression-free survival; OS, overall survival; SCLC, small cell lung cancer. 
Table 3 Occurrence of adverse reactions in SCLC patients

\begin{tabular}{lcc}
\hline Adverse reactions & Grade 1-2 & Grade 3-4 \\
\hline Bone marrow suppression & $6(22.2)$ & $2(7.4)$ \\
Nausea or vomiting & $8(29.6)$ & 0 \\
Proteinuria & $4(14.8)$ & 0 \\
Hypertension & $1(3.7)$ & 0 \\
\hline
\end{tabular}

SCLC, small cell lung cancer.

to those of simple type; after treatment, $13-45 \%$ of simple SCLC showed morphological changes, showing increased cell size and histological characteristics of mixed SCLC.

At present, the platinum-etoposide regimen is the firstline treatment for patients with SCLC, and despite a high remission rate, patients with ES-SCLC often show drug resistance or progress. The median PFS is only 5.5 months and the median OS is less than 10 months. The therapeutic effect remains unsatisfactory following first-line treatment progress, and the 1-year OS rate of topotecan as a second-line therapy is only $30 \%(13-16)$. With the rapid development of immunotherapy; IMpower133, CASPIAN, and other experimental results have shown that immunotherapy combined with standard chemotherapy was able to break through the median OS of SCLC for 12 months, and the NCCN recommended it as a first-line treatment for SCLC in 2019 (17). However, due to the high cost and safety concerns associated with immunotherapy, standard chemotherapy is still the dominant therapeutic option in the clinical treatment of SCLC (18). Therefore, how to treat SCLC has become a particular focus of attention, and there is an urgent need to explore new drugs and treatments with high accessibility.

Anti-angiogenic targeted drugs can inhibit the growth of tumor tissue, but cannot eliminate tumor cells or exert lasting anti-tumor activity. Endostatin is an anti-angiogenic drug that was independently developed in China, and has achieved a certain curative effect in clinical application. The conventional application of endostatin is $7.5 \mathrm{mg} / \mathrm{m}^{2}$, intravenous drip 3-4 hours, once a day, continuous infusion for 14 days. Owing to its short half-life, the blood concentration cannot maintain the effective dose in a routine application, and thus the curative effect is not ideal $(19,20)$. This is due to the fact that malformed blood vessels in the tumor microenvironment can affect the entry of drugs into tumor cells by altering vascular permeability, thereby reducing the curative effect. Endostatin can normalize abnormal blood vessels by changing the growth of vascular endothelium and typically achieves vascular normalization
3-7 days after application. Continuous low-dose antiangiogenic drugs can enhance the efficacy of treatment. Thus, the use of chemotherapeutic drugs after continuous intravenous infusion can promote the entry of drugs into tumor tissue and maximize the efficacy of treatment (21-25). Based on this, continuous intravenous infusion of endostatin combined with chemotherapy was used in this experiment. Since the safe dose of continuous infusion of endostatin is $7.5-30 \mathrm{mg} /\left(\mathrm{m}^{2} \cdot \mathrm{d}\right)(26)$, endostatin $(90 \mathrm{mg})$ was used (via infusion pump) for 72 hours, followed by the infusion of chemotherapeutic drugs. Subsequently, endostatin $(120 \mathrm{mg})$ was continuously injected for 96 hours to maintain a long period of tumor vascular normalization and effective drug concentration, in order to maximize the effectiveness of treatment and play a stronger role of combination.

In this study, 21 SCLC patients treated with endostatin combined with chemotherapy had an ORR of $61.9 \%$, a DCR of $95.2 \%$, a median PFS of 8 months, and a median OS of 13.6 months. At present, the optimal scheme for firstline treatment of SCLC is atezolizumab combined with the platinum-etoposide regimen; the ORR is $60.2 \%$, the median PFS is 5.2 months, and the median OS is 12.3 months (27). As for intravenous infusion of endostatin combined with chemotherapy in the treatment of SCLC, the median PFS is 5 months, the median OS is 11.5 months, and the ORR is $69.7 \%$ (28). The ORR in this study was similar to that of IMpower133, which was lower than that of the intravenous infusion regimen. However, from the perspective of prognostic survival data, the median PFS was significantly prolonged in this study. The longest median OS for treatment for SCLC was 13.0 months (29), and the OS results of this study were similar, with 11 patients $(52.3 \%)$ having an OS of more than 13 months. As of the last follow-up, six patients were still alive, and the longest OS was 30.9 months. Compared with a published study, this study optimized the mode of endostatin administration. Compared with the 14-day intravenous infusion of endostatin, continuous intravenous pump combined chemotherapy can shorten the hospitalization time of patients and improve their compliance and quality of life. At the same time, the purpose of this study is to explore whether the time window of vascular normalization can be established and the curative effect can be improved by optimizing the administration time of antiangiogenic drugs before and after chemotherapy. Also, this study included limited and extensive, treated and newly treated patients with small cell lung cancer, which has wider coverage than the patients in the above study, which provides more 
possibilities for the application of endostatin combined chemotherapy in the treatment of SCLC. In summary, the treatment regimen in this study has some benefits on PFS, and the benefits of PFS could be transformed into OS benefits. Endostatin combined with chemotherapy with an intravenous infusion pump can improve the efficacy of SCLC treatment.

In terms of adverse reactions, the optimal administration regimen in this study was the continuous infusion of $210 \mathrm{mg}$ endostatin via a PICC or port vein infusion pump before and after the application of chemotherapeutic drugs in each treatment cycle. The incidence of grade 2 adverse reaction events was $25 \%$, which mainly involved myelosuppression, nausea, and vomiting. One patient developed grade 4 myelosuppression with thrombocytopenia and recovered after symptomatic treatment. There was no drug reduction or termination of treatment, and no treatment-related death occurred. The common adverse reactions of endostatin combined chemotherapy were hematological toxicity (leukopenia or thrombocytopenia), gastrointestinal reactions (nausea, vomiting), anorexia, elevated transaminase, fatigue, and so on $(30,31)$. In patients with SCLC treated with immunotherapy, the incidence of drug withdrawal due to adverse reactions was $10 \%, 25 \%$ of patients withdrew once due to adverse reactions, and the incidence of severe adverse reactions was $45 \%$ (32). In IMpower133, the incidence of grade 3 or 4 adverse reactions in the combined immunotherapy group was higher than $58.1 \%$, the incidence of reduction due to adverse reactions was $11.1 \%$, and the incidence of immune-related adverse reactions was $39.9 \%$ (27). In comparison, the occurrence of adverse reactions in this experiment was markedly superior to that of immunotherapy, and was similar to the previous endostatin intravenous drip regimen. This may be related to the use of chemotherapeutic drugs after administration of endostatin in advance to rearrange vascular endothelial cells in the tumor microenvironment and the induction and promotion of apoptosis of abnormal tumor vascular endothelial cells in the tumor microenvironment. It is related to the normalization of tumor blood vessels and the increase of the concentration of cytotoxic drugs in the tumor tissue. It can not only enhance the killing effect on tumor cells but also reduce the concentration of cytotoxic drugs in normal tissue, reduce the occurrence of adverse reactions, improve the therapeutic effect. And finally give endostatin again to maintain the stability of blood concentration in the body, prolong the retention time of therapeutic drugs in the body, and further enhance the active components of therapeutic drugs in tumor tissue. Based on this, endostatin combined with chemotherapy not only provides certain survival benefits to SCLC patients, but also has good tolerance and safety. However, there are still adverse reactions, although most of them can be treated with regular monitoring, timely administration of necessary treatment, and so on.

Bevacizumab combined with chemotherapy can improve PFS, but not OS in the treatment of SCLC. The advent of small molecular anti-angiogenesis drugs has made progress in anti-angiogenesis therapy for SCLC. The first-line treatment of advanced SCLC with anlotinib combined with EP regimen shows that the median PFS is 9.61 months, which is twice longer than that of chemotherapy, and the result of OS is not yet mature. Based on the current research, although there is no significant benefit from traditional anti-angiogenesis drugs combined with chemotherapy in the treatment of advanced small cell lung cancer, the emergence of small molecular anti-angiogenesis drugs provides more options for combined therapy. The results of this study also provide a new idea for traditional anti-angiogenesis drugs combined with chemotherapy in the treatment of SCLC. Therefore, whether it can be optimized by anti-angiogenesis drug administration mode, administration time, drug dose, combined application and other aspects, so that the application of anti-angiogenesis therapy in the treatment of SCLC needs further research.

\section{Limitations}

This study has some limitations that should be noted. Firstly, the sample size included in this study was small, there were some limited-stage patients, and the basic state was well. Also, there was no subgroup analysis for different clinical baseline data in the statistical analysis, and thus, some of the results need to be confirmed by phase 3 clinical studies with larger sample sizes. Also, this study is a single-arm multicenter non-blind study, there was no control group, and the curative effect evaluation and other data collection were performed by observers, which can inevitably lead to bias.

\section{Conclusions}

In this study, the median PFS was 8.0 months, the median OS was 13.6 months, the ORR was $61.9 \%$, and the DCR was $95.2 \%$. All patients tolerated the treatment. For the first-line treatment of SCLC, the "sandwich method" intravenous pump infusion of endostatin combined with 
chemotherapy provides a new feasible treatment for SCLC patients. In the follow-up, we can explore the application dose of endostatin, the patients who benefit most, the treatment after progress, maintenance of the treatment dose, and maintenance time, etc. At the same time, we can perform experiments with larger sample sizes to identify the best treatment model and provide new possibilities for the treatment of SCLC.

\section{Acknowledgments}

Funding: None.

\section{Footnote}

Reporting Checklist: The authors have completed the TREND reporting checklist. Available at http://dx.doi. org/10.21037/apm-21-443

Data Sharing Statement: Available at http://dx.doi. org/10.21037/apm-21-443

Conflicts of Interest: All authors have completed the ICMJE uniform disclosure form (available at http://dx.doi. org/10.21037/apm-21-443). The authors have no conflicts of interest to declare.

Ethical Statement: The authors are accountable for all aspects of the work in ensuring that questions related to the accuracy or integrity of any part of the work are appropriately investigated and resolved. All of the enrolled patients or their family members signed the informed consent and could actively cooperate with the treatment. All procedures performed in this study involving human participants were in accordance with the Declaration of Helsinki (as revised in 2013). The study was approved by ethics committee of First Affiliated Hospital of Dalian Medical University (No. YJ-KYFB-2020-23).

Open Access Statement: This is an Open Access article distributed in accordance with the Creative Commons Attribution-NonCommercial-NoDerivs 4.0 International License (CC BY-NC-ND 4.0), which permits the noncommercial replication and distribution of the article with the strict proviso that no changes or edits are made and the original work is properly cited (including links to both the formal publication through the relevant DOI and the license).
See: https://creativecommons.org/licenses/by-nc-nd/4.0/.

\section{References}

1. Chinese Society of Clinical Oncology. Guidelines for the diagnosis and treatment of small cell lung cancer. 2020.

2. Calles A, Aguado G, Sandoval C, et al. The role of immunotherapy in small cell lung cancer. Clin Transl Oncol 2019;21:961-76.

3. Hu J, Wang Y, Zhang Y, et al. Comprehensive genomic profiling of small cell lung cancer in Chinese patients and the implications for therapeutic potential. Cancer Med 2019;8:4338-47.

4. Byers LA, Rudin CM. Small cell lung cancer: where do we go from here? Cancer. 2015;121:664-72.

5. Lu T, Yang X, Huang Y, et al. Trends in the incidence, treatment, and survival of patients with lung cancer in the last four decades. Cancer Manag Res 2019;11:943-53.

6. Turrisi AT 3rd, Kim K, Blum R, et al. Twice-daily compared with once-daily thoracic radiotherapy in limited small-cell lung cancer treated concurrently with cisplatin and etoposide. N Engl J Med 1999;340:265-71.

7. Owonikoko TK, Behera M, Chen Z, et al. A systematic analysis of efficacy of second-line chemotherapy in sensitive and refractory small-cell lung cancer. J Thorac Oncol 2012;7:866-72.

8. Gazdar AF, Bunn PA, Minna JD. Small-cell lung cancer: what we know, what we need to know and the path forward. Nat Rev Cancer 2017;17:725-37.

9. Ramjiawan RR, Griffioen AW, Duda DG. Antiangiogenesis for cancer revisited: Is there a role for combinations with immunotherapy? Angiogenesis 2017;20:185-204.

10. Mamdani H, Induru R, Jalal SI. Novel therapies in small cell lung cancer. Transl Lung Cancer Res 2015;4:533-44.

11. Shang L, Zhao J, Wang W, et al. Inhibitory effect of endostar on lymphangiogenesis in non-small cell lung cancer and its effect on circulating tumor cells. Zhongguo Fei Ai Za Zhi 2014;17:722-9.

12. Rudin CM, Brambilla E, Faivre-Finn C, et al. Small-cell lung cancer. Nat Rev Dis Primers 2021:14;7:3.

13. Rudin CM, Ismaila N, Hann CL, et al. Treatment of Small-Cell Lung Cancer: American Society of Clinical Oncology Endorsement of the American College of Chest Physicians Guideline. J Clin Oncol 2015;33:4106-11.

14. Früh M, De Ruysscher D, Popat S, et al. Small-cell lung cancer (SCLC): ESMO Clinical Practice Guidelines for diagnosis, treatment and follow-up. Ann Oncol 
2013;24:vi99-105.

15. Dómine M, Moran T, Isla D, et al. SEOM clinical guidelines for the treatment of small-cell lung cancer (SCLC) (2019). Clin Transl Oncol 2020;22:245-55.

16. DiBonaventura MD, Shah-Manek B, Higginbottom K, et al. Adherence to recommended clinical guidelines in extensive disease small-cell lung cancer across the US, Europe, and Japan. Ther Clin Risk Manag 2019;15:355-66.

17. NCCN Clinical Practice Guidelines in Oncology (NCCN Guidelines®)Small Cell Lung Cancer (Version 1, 2019).

18. Li LY, Wang H, Chen X, et al. First-line atezolizumab plus chemotherapy in treatment of extensive small cell lung cancer: a cost-effectiveness analysis from China. Chin Med J (Engl) 2019;132:2790-4.

19. Hu W, Fang J, Nie J, et al. Efficacy and safety of extended use of platinum-based doublet chemotherapy plus endostatin in patients with advanced nonsmall cell lung cancer. Medicine (Baltimore) 2016;95:e4183.

20. Jiang XD, Qiao Y, Dai P, et al. Enhancement of recombinant human endostatin on the radiosensitivity of human pulmonary adenocarcinoma A549 cells and its mechanism. J Biomed Biotechnol 2012;2012:301931.

21. Folkman J, Bach M, Rowe JW, et al. Tumor angiogenesistherapeutic implications. N Engl J Med 1971;285:1182-6.

22. Folkman J, Hahnfeldt P, Hlatky L. Cancer: looking outside the genome. Nat Rev Mol Cell Biol 2000;1:76-9.

23. Jain RK. Normalization of tumor vasculature: an emerging concept in antiangiogenic therapy. Science 2005;307:58-62.

24. Lin MI, Sessa WC. Antiangiogenic therapy: creating a unique "window" of opportunity. Cancer Cell 2004;6:529-31.

25. Liu BD, $\mathrm{Hu} M$, Liu L, et al. The vascular normalization window with recombinant human endostatin in non-

Cite this article as: Zhao $\mathrm{Y}$, Zhang $\mathrm{X}$, Jin $\mathrm{C}$, Yu X, Zhang M, Cao Y, Li Y, Wang A, Shan X, Zhang J, Wang J, Yin L, Tan X, Liu J. Efficacy and safety of endostatin in combination with chemotherapy in small cell lung cancer: a phase 2 single-arm multicenter open-label trial. Ann Palliat Med 2021;10(3):3277-3285. doi: 10.21037/apm-21-443 small cell lung cancer. Chinese Journal of the Frontiers of Medical Science (Electronic Version) 2017:9:8-12.

26. Zhou Q, Hu JW, Yin R, et al. Clinical observation of recombinant human endostatin durative transfusion combined with window period chemotherapy in advanced non-small cell lung cancer. Chinese Clinical Oncology 2014:19:1113-7.

27. Horn L, Mansfield AS, Szczęsna A, et al. First-Line Atezolizumab plus Chemotherapy in Extensive-Stage Small-Cell Lung Cancer. N Engl J Med 2018;379:2220-9.

28. Zhou ZT, Zhou FX, Wei Q, et al. Phase II study of cisplatin/etoposide and endostar for extensive-stage small-cell lung cancer. Cancer Chemother Pharmacol 2011;68:1027-32.

29. Paz-Ares L, Dvorkin M, Chen Y, et al. Durvalumab plus platinum-etoposide versus platinum-etoposide in firstline treatment of extensive-stage small-cell lung cancer (CASPIAN): a randomised, controlled, open-label, phase 3 trial. Lancet 2019;394:1929-39.

30. Lu S, Li L, Luo Y, et al. A multicenter, open-label, randomized phase II controlled study of rh-endostatin (Endostar) in combination with chemotherapy in previously untreated extensive-stage small-cell lung cancer. J Thorac Oncol 2015;10:206-11.

31. Gao SR, Li LM, Xia HP, et al. Clinical observation on recombinant human endostatin combined with chemotherapy for advanced gastrointestinal cancer. Asian Pac J Cancer Prev 2015;16:4037-40.

32. Antonia SJ, López-Martin JA, Bendell J, et al. Nivolumab alone and nivolumab plus ipilimumab in recurrent smallcell lung cancer (CheckMate 032): a multicentre, openlabel, phase 1/2 trial. Lancet Oncol 2016;17:883-95.

(English Language Editor: A. Kassem) 